UK-04-06 UCTP-106-04

March 2004 hep-th/0403109

\title{
Resolving the M2-brane
}

\author{
Chiang-Mei Chen ${ }^{\dagger}$ and Justin F. Vázquez-Poritz ${ }^{\ddagger}$ \\ ${ }^{\dagger}$ Department of Physics, \\ National Central University, Chungli 320, Taiwan \\ ${ }^{\ddagger}$ Department of Physics and Astronomy, \\ University of Kentucky, Lexington, KY 40506 \\ $\ddagger$ Department of Physics, \\ University of Cincinnati, Cincinnati OH 45221-0011
}

\begin{abstract}
$\underline{\text { ABSTRACT }}$
We construct deformed, $T^{2}$-wrapped, rotating M2-branes on a resolved cone over $Q^{1,1,1}$ and $Q^{1,1,1} / \mathbb{Z}_{2}$, as well as on a product of two Eguchi-Hanson instantons. All worldvolume directions of these supersymmetric and regular solutions are fibred over the transverse space. These constitute gravity duals of $D=3 N=2$ gauge theories. In particular, the deformed M2-brane on a resolved cone over $Q^{1,1,1}$ and the $S^{1}$ wrapped M2-brane on a resolved cone over $Q^{1,1,1} / \mathbb{Z}_{2}$ provide explicit realizations of holographic renormalization group flows in M-theory for which both conformal and Lorentz symmetries are broken in the IR region and restored in the UV limit. These solutions can be dualized to supersymmetric type IIB pp-waves, which are rendered non-singular either by additional flux or a twisted time-like direction.
\end{abstract}




\section{Introduction}

The AdS/CFT correspondence enables the study of strongly-coupled gauge theories in terms of $p$-brane backgrounds [1, 2, 3. In order to reduce the supersymmetry to a minimum, one can replace the standard flat space that is transverse to the $p$-brane by a space of special holonomy, which is Ricci-flat and has fewer covariantly constant spinors. In order for the solution to be non-singular, the transverse space must have a non-collapsing $n$-cycle and thus support an $n$-form which is square integrable at short distance. As a prelude to resolving the M2-brane, we will briefly review the case of the well-studied D3-brane of type IIB theory.

The simplest example of a six-dimensional Calabi-Yau manifold that can be used for the transverse space is the conifold, defined as a cone over $T^{1,1}=\left(S^{3} \times S^{3}\right) / S^{1}$. In the decoupling limit, the resulting geometry is $\operatorname{AdS}_{5} \times T^{1,1}$, which provides a gravity dual to $\mathcal{N}=1, D=4$ superconformal Yang-Mills theory. Wrapping 5-branes upon a supersymmetric 2-cycle in $T^{1,1}$ gives rise to supersymmetric fractional D3branes [4, 5, 6, 7]. A distance-dependent logarithmic contribution to the D3-brane charge breaks the conformal symmetry. However, this also induces a short-distance naked singularity and therefore provides structural behavior only at large distance, corresponding to the UV region of the dual gauge theory. The above construction involves the complex 3 -form $F_{(3)}=F_{(3)}^{\mathrm{RR}}+i F_{(3)}^{\mathrm{NS}}$, which is set proportional to a selfdual 3-form supported by the conifold. If the conifold has a non-collapsing 3-cycle then the 3-form is square integrable at short distance, resulting in the resolution of the above naked singularity [8].

There are two smooth versions of the conifold, known as the deformed conifold and the resolved conifold [9]. In the former case, the singular apex is blown up to a smooth three-sphere, yielding a non-collapsing 3-cycle. The supersymmetric and regular fractional D3-brane on the deformed conifold was constructed in [5], and provides a geometrical realization of chiral symmetry breaking and confinement. On the other hand, for the resolved conifold, the singular apex is blown up to a smooth two-sphere. Since it has a collapsing 3-cycle, the fractional D3-brane over the resolved conifold has a repulson-like naked singularity [10]. In addition, it is not supersymmetric 11, 12. The same applies to the fractional D3-brane constructed 
with a resolved cone over $T^{1,1} / \mathbb{Z}_{2}$ (also known as a regularized conifold), for which the singular apex has been blown up to a smooth $S^{2} \times S^{2}[13]$.

The non-collapsing 2-cycle of the resolved conifold over $T^{1,1}$ implies that there is a square integrable harmonic 2-form, yielding a regular solution. Since there is no 2 -form field strength in type IIB theory, this contribution must take the form of a fibration, as was originally observed for the D5-brane [14]. Thus, a D3-brane wrapped on $S^{1}$, which is fibred over the resolved conifold, is regular and preserves a minimal amount of supersymmetry [15. In the case of the cone over $T^{1,1} / \mathbb{Z}_{2}$, there is a noncollapsing 4-cycle. The square integrable harmonic 4-form comes about by taking the Hodge dual of a 2 -form in the six-dimensional transverse space. Therefore, there is a regular and supersymmetric $S^{1}$-wrapped D3-brane on the resolved cone over $T^{1,1} / \mathbb{Z}_{2}$. From the viewpoint of the dual gauge theory, both conformal and Lorentz symmetries are broken in the IR region and restored in the UV limit.

It has been conjectured that the fractional D3-brane and $S^{1}$-wrapped D3-brane have a common origin in M-theory as a modified supermembrane on a $\operatorname{Spin}(7)$ manifold, which gives rise to the deformed and resolved conifolds in different GromovHausdorff limits [15]. The $G_{2}$ unification of deformed and resolved conifolds supports this claim [16, 17, 18].

We will now turn to the case of the M2-brane, which has an eight-dimensional transverse space. M2-branes with minimal supersymmetries were first discussed in [19] by replacing the transverse $S^{7}$ with another Einstein space. Supersymmetric and regular deformed M2-branes have been studied extensively, for example, in [8, 11, 12, 20, 21, 22, 23, 24, 25, 26, 27, 28. Singularity resolution of the M2-brane solution due to gravitational Chern-Simons corrections has been considered in 29].

The main goal of this paper is the construction of supersymmetric and regular $S^{1}$-wrapped M2-branes and rotating M2-branes [30]. One might wonder if this can be done indirectly by dualizing the deformed M2-brane. Explicitly dualizing the M2brane to a type IIB pp-wave shows that this is not possible from the type IIB context. On the other hand, one could imagine a unification in higher dimensions, such as Ftheory, analogous to the M-theory unification of fractional and $S^{1}$-wrapped D3-branes. Although the involvement of twelve dimensions is on rather shaky ground, we will briefly consider this at least formally, in the spirit of 31. An immediate objection 
would be that nine-dimensional Ricci-flat manifolds do not have an irreducible special holonomy group under which eight-dimensional manifolds of special holonomy can be unified.

Alternatively, there are eight-dimensional Ricci-flat Kähler spaces which have both non-collapsing 4-cycles and non-collapsing 2-cycles, enabling the properties of a deformed M2-brane and wrapped M2-brane to be combined into one solution. These spaces include the cone over $Q^{1,1,1}$ and $Q^{1,1,1} / \mathbb{Z}_{2}[32$, as well as the product space $M_{4} \times M_{4}$, where each $M_{4}$ is either an Eguchi-Hanson [33] or Taub-NUT instanton [34]. We find a self-dual harmonic four-form and harmonic two-forms which are square integrable at short distance. All of these components can be used to construct a rather general supersymmetric and regular solution. This is a deformed, $T^{2}$-wrapped and rotating M2-brane, since it is modified by additional flux and all worldvolume directions, including time, are fibred over the transverse space.

These solutions are dual to three-dimensional $N=2$ gauge theories. In certain cases, the geometry asymptotically approaches $\mathrm{AdS}_{4} \times Q^{1,1,1}$, the CFT dual of which has been discussed in [24, 35, 36, 37, 38, 39, 40, 41, 42. However, in the present solutions the singularity at the tip of the conifold has been resolved, and the M2brane solution is rendered regular. These particular solutions exhibit holographic renormalization group $(\mathrm{RG})$ flows for which both conformal and Lorentz symmetries are broken in general but are restored in the UV limit. Other RG flows in M-theory which flow from $E^{2,1} \times M_{8}$ at small distance to $A d S_{4} \times M_{7}$ at large distance have been studied in [42, 43], which correspond to $D=3$ super Yang Mills theories of various supersymmetry.

For better clarity, we will first discuss deformed, wrapped and rotating M2-branes separately, before combining them into the general M2-brane solution. This paper is organized as follows. In section 2, we discuss the deformed M2-brane on Ricci-flat Kähler spaces. These M2-brane solutions are supersymmetric and, due to additional flux, regular. In section 3, we discuss $S^{1}$-wrapped or rotating M2-branes, for which the wrapped or time-like coordinate of the worldvolume is fibred over the transverse space. Now it is this fibration, rather than additional flux, which results in the solution being regular. Since the transverse spaces that we are considering support both an appropriate harmonic 4-form and three harmonic 2-forms, we are able to 
construct a rather general M2-brane in section 4. In addition to being deformed by additional flux, this solution is also $T^{2}$-wrapped and rotating. In section 5 , we dualize the general M2-brane to a type IIB supersymmetric pp-wave. The geometry of the pp-wave is non-singular, due to both a variety of fluxes as well as a twisted time-like direction. Similar pp-waves are obtained from wrapped and rotating D3-branes and D5-branes. The conclusions are given in section 6 .

\section{Deformed M2-brane}

The M2-brane of eleven-dimensional supergravity is supported by the 4-form field strength, with an eight-dimensional Ricci-flat transverse space. Due to the equation of motion $d * F_{(4)}=\frac{1}{2} F_{(4)} \wedge F_{(4)}$, one can construct a resolved M2-brane if the transverse space has a (anti)-self-dual 4-cycle. This type of modification to the M2-brane, which makes use of the interaction in $d * F_{(4)}=\frac{1}{2} F_{(4)} \wedge F_{(4)}$, has been greatly studied, for example in [8, 11, 12, 20, 21, 22, 23, 24, 25, 26, 27]. The deformed M2-brane is given by

$$
\begin{aligned}
d s_{11}^{2} & =H^{-2 / 3} d x^{\mu} d x^{\nu} \eta_{\mu \nu}+H^{1 / 3} d s_{8}^{2} \\
F_{(4)} & =d^{3} x \wedge d H^{-1}+m G_{(4)}
\end{aligned}
$$

where $G_{(4)}$ is a harmonic self-dual 4 -form in the Ricci-flat transverse space $d s_{8}^{2}$. The equations of motion are satisfied, provided that

$$
\square H=-\frac{1}{48} m^{2} G_{(4)}^{2},
$$

where $\square$ is the Laplacian on $d s_{8}^{2}$. The introduction of $G_{(4)}$ to the M2-brane solution does not break additional supersymmetries, provided that

$$
G_{a b c d} \Gamma^{b c d} \epsilon=0
$$

where $\epsilon$ is a Killing spinor in the transverse space [23]. The M2-branes discussed in this paper preserve $\frac{1}{4}$ of the original supersymmetry.

\subsection{On the resolved cone over $Q^{1,1,1}$}

For the transverse space, we will first consider an eight-dimensional resolved cone over $Q^{1,1,1}$ (or $Q^{1,1,1} / \mathbb{Z}_{2}$ ) 32 , where the Einstein space $Q^{1,1,1}$ can be expressed as the 
coset 44

$$
Q^{1,1,1}=\frac{S U(2)^{3}}{U(1)^{2}}
$$

The corresponding metric is of the form

$$
d s_{8}^{2}=d \rho^{2}+c^{2} \sigma^{2}+\sum_{i=1}^{3} a_{i}^{2}\left(d \Omega_{2}^{i}\right)^{2} .
$$

where

$$
\left(d \Omega_{2}^{i}\right)^{2}=d \theta_{i}^{2}+\sin ^{2} \theta_{i} d \phi_{i}^{2}, \quad \sigma=d \psi+\sum_{i=1}^{3} \cos \theta_{i} d \phi_{i} .
$$

The existence of Killing spinors implies the following first-order equations:

$$
\dot{c}=1-2 \sum_{i} \dot{a}_{i}^{2}, \quad c=2 a_{i} \dot{a}_{i}
$$

where there is no implicit summation in the second expression. These first-order equations imply that the space is Ricci-flat and Kähler. The resolved eight-dimensional conifold is given by

$$
\begin{aligned}
a_{i}^{2} & =\frac{1}{8}\left(r^{2}+\ell_{i}^{2}\right), \quad c^{2}=\frac{r^{2}}{16 h^{2}}, \quad d \rho=h d r \\
h^{2} & =\frac{3 \prod_{i}\left(r^{2}+\ell_{i}^{2}\right)}{3 r^{6}+4 \sum_{i} \ell_{i}^{2} r^{4}+6 \sum_{i<j} \ell_{i}^{2} \ell_{j}^{2} r^{2}+12 \prod_{i} \ell_{i}^{2}} .
\end{aligned}
$$

This geometry with two non-vanishing $\ell_{i}$ was found in [12, and with three nonvanishing $\ell_{i}$ was found in [8]. In each case, it was derived from the first-order equations which result from the superpotential.

The range of the radial coordinate in is $0 \leq r<\infty$. In order for the geometry to be regular at $r=0$, no more than one $\ell_{i}$ can vanish. For two non-vanishing $\ell_{i}, \psi$ has a period of $4 \pi$ and the principal orbit is $Q^{1,1,1}$. The geometry is $\mathbb{R}^{4} \times S^{2} \times S^{2}$ at small distance and the cone over $Q^{1,1,1}$ asymptotically. Topologically, the manifold is a $\mathbb{C}^{2}$ bundle over $\mathbb{C P}^{1} \times \mathbb{C P}^{1}$.

If all three $\ell_{i}$ are non-vanishing, then the period of $\psi$ is $2 \pi$ and the principal orbit is $Q^{1,1,1} / Z_{2}$. The geometry is $\mathbb{R}^{2} \times S^{2} \times S^{2} \times S^{2}$ at small distance and the cone over $Q^{1,1,1} / \mathbb{Z}_{2}$ asymptotically. Topologically, the manifold is a complex-line bundle over $\mathbb{C P}^{1} \times \mathbb{C P}^{1} \times \mathbb{C P}^{1}$. If all three $\ell_{i}$ are equal, which will be a simple case frequently discussed in this paper, then we can replace $\mathbb{C P}^{1} \times \mathbb{C P}^{1} \times \mathbb{C P}^{1}$ by any other EinsteinKähler 6-space. Note that the regular cone with three non-vanishing $\ell_{i}$ does not 
reduce to the regular cone with two non-vanishing $\ell_{i}$ in the limit that one of the $\ell_{i}$ goes to zero, since the two spaces have different principal orbits.

The veilbein for the 8-space described by (2.5) and (2.8) are given by

$$
e^{0}=h d r, \quad e^{7}=c \sigma, \quad e^{2 j-1}=a_{j} d \theta_{j}, \quad e^{2 j}=a_{j} \sin \theta_{j} d \phi_{j},
$$

for $j=1,2,3$. We construct a harmonic self-dual 4 -form on this 8-space with the ansatz

$$
\begin{gathered}
G_{(4)}=u_{1}\left(e^{0} \wedge e^{7} \wedge e^{1} \wedge e^{2}+e^{3} \wedge e^{4} \wedge e^{5} \wedge e^{6}\right)+u_{2}\left(e^{0} \wedge e^{7} \wedge e^{3} \wedge e^{4}+e^{1} \wedge e^{2} \wedge e^{5} \wedge e^{6}\right) \\
+u_{3}\left(e^{0} \wedge e^{7} \wedge e^{5} \wedge e^{6}+e^{1} \wedge e^{2} \wedge e^{3} \wedge e^{4}\right)
\end{gathered}
$$

The closure of $G_{(4)}$ yields

$$
\begin{aligned}
u_{1} & =\frac{\left(\ell_{2}^{2}+\ell_{3}^{2}\right)\left(r^{4}+\ell_{1}^{2} \ell_{2}^{2}\right)+2 \ell_{2}^{2}\left(\ell_{1}^{2}+\ell_{3}^{2}\right) r^{2}}{\left(r^{2}+\ell_{1}^{2}\right)\left(r^{2}+\ell_{2}^{2}\right)^{2}\left(r^{2}+\ell_{3}^{2}\right)^{2}} \\
u_{2} & =-\frac{\left(\ell_{1}^{2}+\ell_{3}^{2}\right)\left(r^{4}+\ell_{1}^{2} \ell_{2}^{2}\right)+2 \ell_{1}^{2}\left(\ell_{2}^{2}+\ell_{3}^{2}\right) r^{2}}{\left(r^{2}+\ell_{1}^{2}\right)^{2}\left(r^{2}+\ell_{2}^{2}\right)\left(r^{2}+\ell_{3}^{2}\right)^{2}} \\
u_{3} & =\frac{\left(\ell_{1}^{2}-\ell_{2}^{2}\right)\left(r^{4}-\ell_{1}^{2} \ell_{2}^{2}\right)}{\left(r^{2}+\ell_{1}^{2}\right)^{2}\left(r^{2}+\ell_{2}^{2}\right)^{2}\left(r^{2}+\ell_{3}^{2}\right)}
\end{aligned}
$$

For $\ell_{1}=0$, this reduces to the harmonic 4 -form given in 12 . There is no charge associated with $G_{(4)}$. Note that the above three $u_{i}$ 's satisfy the following linear relation:

$$
u_{0}+u_{1}+u_{2}=0
$$

which is a necessary, but not sufficient, condition for supersymmetry. In particular, the supersymmetry condition (2.3) implies (2.12) but there are clearly four-forms which satisfy the latter condition without giving rise to supersymmetric solutions.

Since the general solution for $H$ is quite complicated, we will focus on particular cases. For equal $\ell_{i} \equiv \ell$, we find that

$$
\begin{aligned}
H= & c_{0}+\frac{m^{2}}{\ell^{4}\left(r^{2}+\ell^{2}\right)}+\frac{m^{2}+c_{1}}{2 \ell^{6}} \arctan \left(\frac{r^{2}+\ell^{2}}{\ell^{2}}\right) \\
& +\frac{m^{2}-c_{1}}{4 \ell^{6}} \log \left(\frac{r^{2}}{r^{2}+2 \ell^{2}}\right)
\end{aligned}
$$

where $c_{0}$ and $c_{1}$ are arbitrary integration constants. In general, there is a naked singularity as $r \rightarrow 0$. However, we can choose $c_{1}=m^{2}$ such that the logarithmic term 
cancels, leaving a non-singular solution given by

$$
H=c_{0}+\frac{m^{2}}{\ell^{4}\left(r^{2}+\ell^{2}\right)}+\frac{m^{2}}{\ell^{6}} \arctan \left(\frac{r^{2}+\ell^{2}}{\ell^{2}}\right) .
$$

We define $c_{0}$ so that $H$ asymptotically approaches unity at large $r$. Also, this solution, as do all of the solutions presented, has a well-defined ADM mass. The geometry interpolates between $E^{4,1} \times S^{2} \times S^{2} \times S^{2}$ at small $r$ to $E^{2,1}$ and a conifold at large $r$. Since the geometry does not asymptotically approach $\mathrm{AdS}_{4}$ times a compact space at large distance, a dual gauge theory would not have conformal symmetry.

For the case of vanishing $\ell_{1}$, a regular solution is given by

$$
\begin{aligned}
H= & c_{0}-\frac{3 m^{2}\left(\ell_{2}^{2}+\ell_{3}^{2}+3 r^{2}\right)}{2\left(2 \ell_{3}^{2}-\ell_{2}^{2}\right)\left(2 \ell_{2}^{2}-\ell_{3}^{2}\right)\left(r^{2}+\ell_{2}^{2}\right)\left(r^{2}+\ell_{3}^{2}\right)} \\
& +\frac{27 \sqrt{2} m^{2}}{4\left(2 \ell_{3}^{2}-\ell_{2}^{2}\right)^{3 / 2}\left(2 \ell_{2}^{2}-\ell_{3}^{2}\right)^{3 / 2}} \arctan \left[\frac{\sqrt{2\left(2 \ell_{3}^{2}-\ell_{2}^{2}\right)\left(2 \ell_{2}^{2}-\ell_{3}^{2}\right)}}{3 r^{2}+2\left(\ell_{2}^{2}+\ell_{3}^{2}\right)}\right]
\end{aligned}
$$

This latter solution has already been found in [12]. For small $r$, the geometry becomes $E^{6,1} \times S^{2} \times S^{2}$. For large $r$, and for an appropriate value of $c_{0}$, the geometry asymptotically approaches $\mathrm{AdS}_{4} \times Q^{1,1,1}$. This provides an explicit realization of a holographic renormalization group ( $R G$ ) flow in M-theory for which both conformal and Lorentz symmetries are broken in general but are restored in the UV limit of the dual $D=3 N=2$ gauge theory. It is conjectured that, since the M2-branes are coincident, the dual gauge theory is on the Higgs branch [12. In general, the fact that there is no charge associated with $G_{(4)}$ implies that there are no fractional branes. This indicates that the dual gauge theory is perturbed by relevant operators 24 .

The M2-brane (2.1) can be dimensionally reduced over the transverse angular directions to give a $D=4$ domain-wall solution, whose metric is given by

$$
d s_{4}^{2}=H^{1 / 2} \prod_{i} a_{i}^{2} c\left(d x^{\mu} d x^{\nu} \eta_{\mu \nu}+H h^{2} d r^{2}\right)
$$

where the various functions are given in (2.8). For vanishing $\ell_{1}, H$ is given by (2.15) and the domain-wall metric asymptotically approaches $\mathrm{AdS}_{4}$ at large $r$. This implies that the solution is supported by a non-trivial scalar potential in $D=4$ with a fixed point, which corresponds to the holographic renormalization group flow of the dual field theory [12]. 


\subsection{On $M_{4} \times M_{4}$}

We can use a product of two Ricci-flat Kähler 4-spaces, such as the Eguchi-Hanson instanton [33] and Taub-NUT instanton [34, for the transverse space. We will consider two Eguchi-Hanson instantons, for which the transverse metric is given by

$$
d s_{8}^{2}=d s_{4}^{2}+d \tilde{s}_{4}^{2}
$$

where

$$
\begin{aligned}
d s_{4}^{2} & =W^{-1} d r^{2}+\frac{1}{4} r^{2} W(d \psi+\cos \theta d \phi)^{2}+\frac{1}{4} r^{2}\left(d \theta^{2}+\sin ^{2} \theta d \phi^{2}\right) \\
W & =1-\frac{a^{4}}{r^{4}}
\end{aligned}
$$

and likewise for $d \tilde{s}_{4}^{2}$. The radial coordinate $r$ lies in the range $a \leq r \leq \infty$ and $\psi$ has the period $2 \pi$. The geometry is asymptotically locally Euclidean, and has level surfaces of $S^{3} / \mathbb{Z}_{2}$ at constant $r$. The veilbein for the 8-space (2.17) are given by

$$
e^{0}=W^{-1 / 2} d r, \quad e^{1}=\frac{1}{2} r d \theta, \quad e^{2}=\frac{1}{2} r \sin \theta d \phi, \quad e^{3}=\frac{1}{2} r W^{1 / 2}(d \psi+\cos \theta d \phi),
$$

and likewise for $\tilde{e}^{i}$.

The Eguchi-Hanson metric $d s_{4}^{2}$ has a Kähler form given by

$$
J_{(2)}=\left(e^{0} \wedge e^{3}-e^{1} \wedge e^{2}\right)
$$

and likewise for for $d \tilde{s}_{4}^{2}$ and $\tilde{J}_{(2)}$. The Kähler form for the 8-space (2.17) is given by

$$
J=J_{(2)}+\tilde{J}_{(2)}
$$

Also, $d s_{4}^{2}$ supports a self-dual harmonic 2-form given by

$$
L_{(2)}=\frac{2}{r^{4}}\left(e^{0} \wedge e^{3}+e^{1} \wedge e^{2}\right)
$$

and likewise for $d \tilde{s}_{4}^{2}$ and $\tilde{L}_{(2)}$.

An anti-self-dual harmonic 4-form on the 8-space (2.17) can be built out of the Kähler forms and harmonic 2-forms on the individual Eguchi-Hanson spaces, given by

$$
G_{(4)}=L_{(2)} \wedge \tilde{J}_{(2)}+\tilde{L}_{(2)} \wedge J_{(2)}
$$


The equation for $H(2.2)$ becomes

$$
\left(\frac{1}{r^{3}} \partial_{r} r^{3} W \partial_{r}+\frac{1}{\tilde{r}^{3}} \partial_{\tilde{r}} \tilde{r}^{3} \tilde{W} \partial_{\tilde{r}}\right) H=-8 m^{2}\left(\frac{1}{r^{8}}+\frac{1}{\tilde{r}^{8}}\right)
$$

For appropriate integration constants, a regular solution is given by

$$
H=1+\frac{m^{2}}{a^{4} r^{2}}+\frac{m^{2}}{\tilde{a}^{4} \tilde{r}^{2}}
$$

The M2-brane geometry goes from $E^{6,1} \times S^{2} \times S^{2}$ at short distance to $E^{10,1}$ at large distance. If one drops the 1 in the above $H$, then the solution is a domain-wall at large distance.

For the eight-dimensional transverse space given by the metric (2.17), we could also consider the product of two Taub-NUT instantons, as well as the product of an Eguchi-Hanson and Taub-NUT instanton. However, one has to take care that the spaces are oriented such that both of the normalizable harmonic two-forms are either both self-dual or anti-self-dual. This ensures that cross-terms in functions of $r$ and $\tilde{r}$ cancel out in $G_{(4)}^{2}$, such that $H$ can easily be found in the simple form $H=f(r)+g(\tilde{r})$.

\section{Wrapped or rotating M2-brane}

If the transverse space has a 2-cycle $L_{(2)}$, we can construct an $S^{1}$-wrapped M2-brane with one of the world-volume coordinates fibred over the transverse space. Using the same technique developed in [14, 15, 30], we find that the solution is given by

$$
\begin{aligned}
d s_{11}^{2} & =H^{-2 / 3}\left(-d t^{2}+d x_{1}^{2}+\left(d x_{2}+A_{(1)}\right)^{2}\right)+H^{1 / 3} d s_{8}^{2} \\
F_{(4)} & =d t \wedge d x_{1} \wedge\left(d x_{2}+A_{(1)}\right) \wedge d H^{-1}+m d t \wedge d x_{1} \wedge L_{(2)}
\end{aligned}
$$

where $d A_{(1)}=m L_{(2)}$ and $L_{(2)}$ is a harmonic 2-form in the transverse space of the metric $d s_{8}^{2}$. The equations of motion are satisfied if the function $H$ is given by

$$
\square H=-\frac{1}{4} m^{2} L_{(2)}^{2},
$$

where $\square$ is the Laplacian on $d s_{8}^{2}$.

Alternatively, a rotating M2-brane solution is given by

$$
\begin{aligned}
d s_{11}^{2} & =H^{-2 / 3}\left(-\left(d t+A_{(1)}\right)^{2}+d x_{1}^{2}+d x_{2}^{2}\right)+H^{1 / 3} d s_{8}^{2} \\
F_{(4)} & =\left(d t+A_{(1)}\right) \wedge d x_{1} \wedge d x_{2} \wedge d H^{-1}+m d x_{1} \wedge d x_{2} \wedge L_{(2)}
\end{aligned}
$$


where again we require the function $H$ to satisfy (3.2) [30]. Although the rotating M2-brane (3.3) is related to the $S^{1}$-wrapped M2-brane (3.1) by Wick rotations, this involves the analytical continuation $m \rightarrow i m$ which results in a sign change in (3.2). Although this can be counteracted by changing the orientation of the conifold, reversing the orientation of the transverse space can render the solution non-supersymmetric.

\subsection{On the resolved conifold over $Q^{1,1,1}$}

A general two-form on the 8-space given by (2.5) and (2.8) can be expressed in terms of the veilbein as

$$
L_{(2)}=u_{0} e^{0} \wedge e^{7}+u_{1} e^{1} \wedge e^{2}+u_{2} e^{3} \wedge e^{4}+u_{3} e^{5} \wedge e^{6}
$$

where $u_{i}$ are function of $r$ only. The Kähler form is given by

$$
J_{(2)}=-e^{0} \wedge e^{7}+e^{1} \wedge e^{2}+e^{3} \wedge e^{4}+e^{5} \wedge e^{6} .
$$

Its contribution to the function $H$ is badly behaved at large distance and produces an unresolvable naked singularity. Also, it breaks supersymmetry. Therefore, we will not consider the contributions to $H$ from the Kähler form.

In addition to the Kähler form, there are three harmonic 2-forms. The first, which carries non-trivial flux, is given by

$$
\begin{aligned}
& u_{0}=\frac{\alpha\left(r^{2}+\ell_{3}^{2}\right)^{2}+8 \beta\left(r^{2}+\ell_{3}^{2}\right)+3 \alpha \beta}{\left(r^{2}+\ell_{1}^{2}\right)^{2}\left(r^{2}+\ell_{2}^{2}\right)^{2}}, \\
& u_{1}=\frac{2\left(r^{2}+\ell_{3}^{2}\right)^{2}+3 \alpha\left(r^{2}+\ell_{3}^{2}\right)+6 \beta}{\left(r^{2}+\ell_{1}^{2}\right)^{2}\left(r^{2}+\ell_{2}^{2}\right)} \\
& u_{2}=\frac{2\left(r^{2}+\ell_{3}^{2}\right)^{2}+3 \alpha\left(r^{2}+\ell_{3}^{2}\right)+6 \beta}{\left(r^{2}+\ell_{1}^{2}\right)\left(r^{2}+\ell_{2}^{2}\right)^{2}} \\
& u_{3}=-\frac{4\left(r^{2}+\ell_{3}^{2}\right)+3 \alpha}{\left(r^{2}+\ell_{1}^{2}\right)\left(r^{2}+\ell_{2}^{2}\right)}
\end{aligned}
$$

where $\alpha=\ell_{1}^{2}+\ell_{2}^{2}-2 \ell_{3}^{2}$ and $\beta=\ell_{1}^{2} \ell_{2}^{2}+\ell_{3}^{4}-\ell_{1}^{2} \ell_{3}^{2}-\ell_{2}^{2} \ell_{3}^{2}$.

Since it is difficult to solve for the most general $H$ from (3.2), we will consider a few specific cases for which the solution is regular. For $\ell_{3}=0$,

$$
H=c_{0}+\frac{3 m^{2}\left(\ell_{1}^{2}-3 \ell_{2}^{2}\right)^{2}}{8\left(\ell_{1}^{2}-\ell_{2}^{2}\right)\left(\ell_{1}^{2}-2 \ell_{2}^{2}\right)\left(r^{2}+\ell_{1}^{2}\right)}+\frac{3 m^{2}\left(\ell_{2}^{2}-3 \ell_{1}^{2}\right)^{2}}{8\left(\ell_{2}^{2}-\ell_{1}^{2}\right)\left(\ell_{2}^{2}-2 \ell_{1}^{2}\right)\left(r^{2}+\ell_{2}^{2}\right)}
$$




$$
-\frac{27 m^{2}\left(\ell_{1}^{2}-\ell_{2}^{2}\right)^{2}}{8\left(\ell_{1}^{2}-2 \ell_{2}^{2}\right)\left(\ell_{2}^{2}-2 \ell_{1}^{2}\right) \sqrt{2\left(\ell_{1}^{2}-2 \ell_{2}^{2}\right)\left(\ell_{2}^{2}-2 \ell_{1}^{2}\right)}} \arctan \left[\frac{3 r^{2}+2 \ell_{1}^{2}+2 \ell_{2}^{2}}{\sqrt{2\left(\ell_{1}^{2}-2 \ell_{2}^{2}\right)\left(\ell_{2}^{2}-2 \ell_{1}^{2}\right)}}\right],
$$

where the integration constant $c_{0}$ is chosen such that $H$ asymptotically approaches unity for large $r$.

For the case of all equal $\ell_{i} \equiv \ell$, we find that a regular $H$ is given by

$$
H=c_{0}-\frac{3 m^{2}}{2 \ell^{2}} \arctan \left(\frac{r^{2}+\ell^{2}}{\ell^{2}}\right) .
$$

The second harmonic 2-form also carries non-trivial flux and is given by

$$
\begin{aligned}
& u_{0}=\frac{2\left(\ell_{2}^{2}-\ell_{3}^{2}\right)\left(r^{2}+\ell_{3}^{2}\right)^{2}+8 \beta\left(r^{2}+\ell_{3}^{2}\right)+[3 \alpha+\tilde{\alpha}] \beta}{\left(r^{2}+\ell_{1}^{2}\right)^{2}\left(r^{2}+\ell_{2}^{2}\right)^{2}}, \\
& u_{1}=\frac{2\left(\ell_{2}^{2}-\ell_{3}^{2}\right)\left(r^{2}+\ell_{3}^{2}\right)+4 \beta}{\left(r^{2}+\ell_{1}^{2}\right)^{2}\left(r^{2}+\ell_{2}^{2}\right)} \\
& u_{2}=\frac{4\left(r^{2}+\ell_{3}^{2}\right)^{2}+[5 \alpha-\tilde{\alpha}]\left(r^{2}+\ell_{3}^{2}\right)+8 \beta}{\left(r^{2}+\ell_{1}^{2}\right)\left(r^{2}+\ell_{2}^{2}\right)^{2}} \\
& u_{3}=-\frac{4\left(r^{2}+\ell_{3}^{2}\right)+[3 \alpha+\tilde{\alpha}]}{\left(r^{2}+\ell_{1}^{2}\right)\left(r^{2}+\ell_{2}^{2}\right)}
\end{aligned}
$$

where $\tilde{\alpha}=\ell_{1}^{2}-\ell_{2}^{2}$. A regular solution for $\ell_{3}=0$ is given by

$$
\begin{gathered}
H=c_{0}+\frac{3 m^{2} \ell_{2}^{4}}{2\left(\ell_{1}^{2}-\ell_{2}^{2}\right)\left(\ell_{1}^{2}-2 \ell_{2}^{2}\right)\left(r^{2}+\ell_{1}^{2}\right)}+\frac{3 m^{2}\left(\ell_{2}^{2}-2 \ell_{1}^{2}\right)}{2\left(\ell_{2}^{2}-\ell_{1}^{2}\right)\left(r^{2}+\ell_{2}^{2}\right)} \\
-\frac{3 m^{2}\left(\ell_{2}^{2}-2 \ell_{1}^{2}\right)}{2\left(\ell_{1}^{2}-2 \ell_{2}^{2}\right) \sqrt{2\left(\ell_{1}^{2}-2 \ell_{2}^{2}\right)\left(\ell_{2}^{2}-2 \ell_{1}^{2}\right)}} \arctan \left(\frac{3 r^{2}+2 \ell_{1}^{2}+2 \ell_{2}^{2}}{\sqrt{2\left(\ell_{1}^{2}-2 \ell_{2}^{2}\right)\left(\ell_{2}^{2}-2 \ell_{1}^{2}\right)}}\right) .
\end{gathered}
$$

For the case of all equal $\ell_{i} \equiv \ell$, we find that a regular $H$ has the same form as (3.8) with $m \rightarrow 2 m / \sqrt{3}$.

The last harmonic 2-form has vanishing flux, namely

$$
\int_{r \rightarrow \infty} L_{(2)}=0 .
$$

This 2-form is given by

$$
\begin{aligned}
& u_{0}=\frac{3 r^{4}+2 \sum_{i} \ell_{i}^{2} r^{2}+\sum_{i \neq j} \ell_{i}^{2} \ell_{j}^{2}}{\left(r^{2}+\ell_{1}^{2}\right)^{2}\left(r^{2}+\ell_{2}^{2}\right)^{2}\left(r^{2}+\ell_{3}^{2}\right)^{2}}, \\
& u_{1}=\frac{1}{\left(r^{2}+\ell_{1}^{2}\right)^{2}\left(r^{2}+\ell_{2}^{2}\right)\left(r^{2}+\ell_{3}^{2}\right)}, \\
& u_{2}=\frac{1}{\left(r^{2}+\ell_{1}^{2}\right)\left(r^{2}+\ell_{2}^{2}\right)^{2}\left(r^{2}+\ell_{3}^{2}\right)}, \\
& u_{3}=\frac{1}{\left(r^{2}+\ell_{1}^{2}\right)\left(r^{2}+\ell_{2}^{2}\right)\left(r^{2}+\ell_{3}^{2}\right)^{2}} .
\end{aligned}
$$


The resulting solution is only regular if all $\ell_{i}$ are non-vanishing.

For the case of all equal $\ell_{i} \equiv \ell$, we find that a regular $H$ is given by

$$
H=1+\frac{m^{2}}{8 \ell^{8}\left(r^{2}+\ell^{2}\right)^{3}} .
$$

The previous solutions of this section have geometries which interpolate between a product space of $E^{3,1}$ and a $U(1)$ bundle over $S^{2} \times S^{2} \times S^{2}$ at short distance to $E^{2,1}$ and a conifold at large distance. Therefore, if there is a dual gauge theory, then it does not have a phase for which conformal symmetry is restored. On the other hand, the solution given in (3.13) is particularly interesting since, if we drop the 1, the metric interpolates from a product space of $E^{1,1}$ and a $U(1)$ bundle over $\mathbb{R}^{2} \times S^{2} \times S^{2} \times S^{2}$ at short distance to $A d S_{4} \times Q^{1,1,1} / \mathbb{Z}_{2}$ at large distance. As with the deformed M2-brane on a cone over $Q^{1,1,1}$, this solution exhibits a holographic renormalization group (RG) flow for which both conformal and Lorentz symmetries are broken in general but are restored in the UV limit of the dual $D=3 N=2$ gauge theory. Since there is no charge associated with the 4 -form term which arises from the 2 -form (13.12), this indicates that the dual gauge theory is perturbed by relevant operators [24].

Note that, for all three harmonic 2-forms, the four $u_{i}$ 's satisfy the following linear relation:

$$
u_{0}-u_{1}-u_{2}-u_{3}=0
$$

This is a necessary, but not sufficient, condition for supersymmetry. For example, the Kähler form satisfies the above relation without satisfying the supersymmetry equation (2.3).

\subsection{On $M_{4} \times M_{4}$}

The 2-form $L_{(2)}$, given by (2.22), is not only harmonic on the Eguchi-Hanson metric (2.18) but on the eight-dimensional metric (2.17) as well. Likewise for the 2-form $\tilde{L}_{(2)}$. Therefore, we can construct the following harmonic 2-form on (2.17):

$$
G_{(2)}=L_{(2)}+\tilde{L}_{(2)}
$$

For appropriate integration constants, this yields a regular $H$ given by (2.25) with $m \rightarrow m / \sqrt{2}$. As with the deformed M2-brane, we can also find a regular wrapped 
or rotating M2-brane on a product of two Taub-NUT instantons or a product of an Eguchi-Hanson and Taub-NUT instantons.

\section{General M2-brane}

A deformed, $T^{2}$-wrapped, rotating M2-brane on a resolved conifold over $Q^{1,1,1} / \mathbb{Z}_{2}$ can be constructed which contains all of the elements of the previous two sections, by using both the harmonic 4-form and the harmonic 2-forms. An analogous M2-brane can also be constructed on $M_{4} \times M_{4}$, where each $M_{4}$ is either an Eguchi-Hanson or Taub-NUT space. However, the latter case only gives us two harmonic two-forms at our disposal, which means we must decide between either a deformed, $T^{2}$-wrapped (non-rotating) M2-brane or a deformed, $S^{1}$-wrapped, rotating M2-brane. All of these general M2-branes are supersymmetric and regular.

This is the only known cases of a deformed and wrapped/rotating $p$-brane solution which is supersymmetric and regular. For example, although both fractional D3branes and wrapped D3-branes have been constructed, in order to be supersymmetric and regular, different transverse spaces are required. The fractional D3-brane requires a deformed conifold which supports a harmonic 3-form while the wrapped (or rotating) D3-brane requires a resolved conifold which supports harmonic 2-forms. However, a supersymmetric and regular $T^{2}$-wrapped and $S^{1}$-wrapped rotating D3-brane can be constructed from two independent harmonic 2-forms supported by the resolved conifold, which we will discuss.

In the case of the D5-brane, four-dimensional transverse spaces such as the EguchiHanson and Taub-NUT instantons only support a single harmonic 2-form for which the solution is supersymmetric and regular solution. Multi-instanton spaces are expected to support multiple independent 2-forms which would presumably give rise to deformed, wrapped, rotating D5-branes which are supersymmetric and regular. These have yet to be constructed. Also, a Schwarzschild instanton does support two independent harmonic 2-forms but while the resulting D5-brane solutions are regular, they are not supersymmetric. 
A deformed, $T^{2}$-wrapped, rotating M2-brane is given by

$$
\begin{aligned}
d s_{11}^{2} & =H^{-2 / 3} d \tilde{x}^{\mu} d \tilde{x}^{\nu} \eta_{\mu \nu}+H^{1 / 3} d s_{8}^{2}, \\
F_{(4)} & =d^{3} \tilde{x} \wedge d H^{-1}+m_{4} G_{(4)}+\sum_{i \neq j \neq k} m_{k} d \tilde{x}^{i} \wedge d \tilde{x}^{j} \wedge L_{(2)}^{k},
\end{aligned}
$$

where $d \tilde{x}^{i} \equiv d x^{i}+A_{(1)}^{i}$ and $i, j, k=0,1,2$. As an example, we will consider the transverse space to be the resolved conifold over $Q^{1,1,1} / \mathbb{Z}_{2} \cdot G_{(4)}$ is the harmonic 4-

form found in section 2.1. $d A_{(1)}^{i}=m_{i} L_{(2)}^{i}$ ( $i$ not summed), where $L_{(2)}^{i}$ are the three harmonic 2-forms found in section 2.1. The equations of motion are satisfied, provided that

$$
\square H=-\frac{1}{48} m_{4}^{2} G_{(4)}^{2}-\frac{1}{4} \sum_{i=0}^{2} m_{i}^{2}\left(L_{(2)}^{i}\right)^{2} .
$$

We consider the case of all equal $\ell_{i} \equiv \ell$. A regular $H$ is given by

$$
H=c_{0}+\frac{m_{4}^{2}}{\ell^{4}\left(r^{2}+\ell^{2}\right)}+\frac{m_{0}^{2}}{8 \ell^{8}\left(r^{2}+\ell^{2}\right)^{3}}+\left(\frac{m_{4}^{2}}{\ell^{6}}-\frac{3 m_{1}^{2}}{2 \ell^{2}}-\frac{2 m_{2}^{2}}{\ell^{2}}\right) \arctan \left(\frac{r^{2}+\ell^{2}}{\ell^{2}}\right),
$$

which is just a superposition of individual $H$ for each harmonic forms. The roles of $m_{0}, m_{1}$ and $m_{2}$ are, of course, interchangeable. Likewise, a general M2-brane can be constructed on the product of Eguchi-Hanson or Taub-NUT instantons. For the case of two Eguchi-Hanson instantons, the resulting regular $H$ has the same form as in (2.25).

\section{$5 \quad$ Resolved pp-waves}

\subsection{From M2-branes}

The general M2-brane given by (4.1) can be dimensionally reduced on $x_{2}$ to give a deformed, wrapped, rotating NS-NS string in type IIA theory, given by

$$
\begin{aligned}
d s_{10}^{2} & =H^{-3 / 4}\left(-d \tilde{x}_{0}^{2}+d \tilde{x}_{1}^{2}\right)+H^{1 / 4} d s_{8}^{2}, \\
F_{(4)} & =m_{4} G_{(4)}+m_{2} d \tilde{x}_{0} \wedge d \tilde{x}_{1} \wedge L_{(2)}^{2}, \\
F_{(3)} & =d \tilde{x}_{0} \wedge d \tilde{x}_{1} \wedge d H^{-1}+m_{0} d \tilde{x}_{1} \wedge L_{(2)}^{0}+m_{1} d \tilde{x}_{0} \wedge L_{(2)}^{1}, \\
F_{(2)} & =m_{2} L_{(2)}^{2}, \quad e^{2 \phi}=H .
\end{aligned}
$$


Hopf T-duality on the $x_{1}$ coordinate has the effect of untwisting this direction [45]. At the same time, the first two terms in the above 3-form flux are dualized to contributions to the metric. The result is a supersymmetric and regular type IIB pp-wave given by

$$
\begin{aligned}
d s_{10}^{2} & =-H^{-1} d \tilde{x}_{0}^{2}+H\left(d x+B_{(1)}\right)^{2}+d s_{8}^{2}, \\
F_{(5)} & =m_{4}\left(d x+B_{(1)}\right) \wedge\left(G_{(4)}+*_{8} G_{(4)}\right), \\
F_{(3)}^{\mathrm{RR}} & =m_{2}\left(d x+d \tilde{x}_{0}+B_{(1)}\right) \wedge L_{(2)}^{2} \\
F_{(3)}^{\mathrm{NS}} & =m_{1}\left(d x+d \tilde{x}_{0}+B_{(1)}\right) \wedge L_{(2)}^{1},
\end{aligned}
$$

where $\left(H^{-1}-1\right) d \tilde{x}_{0}$. If instead we had reduced the M2-brane over $x_{1}$ and Hopf T-dualized over $x_{2}$, then we would have ended up with the S-dual of the pp-wave solution (5.2), with $F_{(3)}^{\mathrm{RR}} \leftrightarrow F_{(3)}^{\mathrm{NS}}$.

This general solution encompasses various limits in which one or more of the fields vanish, which are related to deformed, wrapped or rotating M2-branes. The connection between deformed M2-branes and supersymmetric type IIB pp-waves has been discussed in [46]. As long as all of the fields $L_{(2)}^{i}$ and $G_{(4)}$ do not vanish simultaneously, the solution (5.2) is completely regular. In particular, vanishing $L_{(2)}^{1}, L_{(2)}^{2}$ and $G_{(4)}$ yields a Ricci-flat pp-wave, whose twisted time-like direction ensures a non-singular geometry.

Finally, for vanishing $L_{(2)}^{2}$ and $G_{(4)}$, (5.1) is also a solution of type IIB theory, which can be S-dualized to a wrapped, rotating D1-brane. This solution can then be Hopf T-dualized to a (smeared) rotating D0-brane. All of these solutions are supersymmetric and regular.

Note that this is more fortunate than the situation for the deformed D1-brane on the conifold, which has a singular geometry. This is because the transverse space does not have a non-collapsing 5-cycle at short distance, and therefore cannot admit an appropriate 5 -form [8, 44]. 


\subsection{From D3-branes}

An $S^{1}$-wrapped rotating D3-brane is given by [15]

$$
\begin{aligned}
d s_{10}^{2}= & H^{-1 / 2}\left(-\left(d t+A_{(1)}^{0}\right)^{2}+d x_{1}^{2}+d x_{2}^{2}+\left(d x_{3}+A_{(1)}^{1}\right)^{2}\right)+H^{1 / 2} d s_{6}^{2} \\
F_{(5)}= & \left(d t+A_{(1)}^{0}\right) \wedge d x_{1} \wedge d x_{2} \wedge\left(d x_{3}+A_{(1)}^{1}\right) \wedge d H^{-1} \\
& +m_{0} *_{6} L_{(2)}^{0} \wedge\left(d t+A_{(1)}^{0}\right)+m_{1} *_{6} L_{(2)}^{1} \wedge\left(d x_{3}+A_{(1)}^{1}\right)+\text { dual terms }
\end{aligned}
$$

where $d A_{(1)}^{i}=m_{i} L_{(2)}^{i}$ for $i=0,1 . L_{(2)}^{i}$ are two independent harmonic 2-forms on $d s_{6}^{2}$, and $*_{6}$ is the Hodge dual with respect to $d s_{6}^{2}$. The equations of motion are satisfied, provided that

$$
\square H=-\frac{1}{2} \sum_{i=0}^{1} m_{i}^{2}\left(L_{(2)}^{i}\right)^{2},
$$

where $\square$ is the Laplacian on $d s_{6}^{2}$. A regular and supersymmetric solution exists for the case in which $d s_{6}^{2}$ is the metric for the resolved conifold on $T^{1,1} / Z_{2}$. For the case in which the D3-brane is not rotating, the metric interpolates between a product of $E^{2,1}$ and a $U(1)$ bundle over $\mathbb{R}^{2} \times S^{2} \times S^{2}$ at short distance to $\operatorname{AdS}_{5} \times T^{1,1} / \mathbb{Z}_{2}$ at large distance. This provides a regular gravity dual to a certain four-dimensional gauge theory whose Lorentz and conformal symmetries are broken in the IR region and restored in the UV limit [15].

Multiple T-dualities yields a type IIB pp-wave, which is given by

$$
\begin{aligned}
d s_{10}^{2} & =-H^{-1}\left(d t+A_{(1)}^{0}\right)^{2}+H\left(d x+\left(H^{-1}-1\right)\left(d t+A_{(1)}^{0}\right)\right)^{2}+d s_{6}^{2}+d z_{1}^{2}+d z_{2}^{2} \\
F_{(5)} & =m_{1} *_{6} L_{(2)}^{1} \wedge d x+m_{1} d z_{1} \wedge d z_{2} \wedge L_{(2)}^{1}+\text { dual terms }
\end{aligned}
$$

Notice that, for vanishing $L_{(2)}^{1}$, the solution is Ricci-flat, for which the twisted time-like direction ensures that the geometry is regular.

Alternatively, we could consider a $T^{2}$-wrapped D3-brane, which dualizes to a type IIB pp-wave given by

$$
\begin{aligned}
d s_{10}^{2} & =-2 d t d x+H d x^{2}+d s_{6}^{2}+d z_{1}^{2}+d z_{2}^{2} \\
F_{(5)} & =m_{1} *_{6} L_{(2)}^{1} \wedge d x+m_{1} d z_{1} \wedge d z_{2} \wedge L_{(2)}^{1}+\text { dual terms } \\
F_{(3)}^{\mathrm{RR}} & =m_{0}(d x+d t) \wedge L_{(2)}^{0} .
\end{aligned}
$$

Dualizing the fibred directions in reverse order results in the S-dual of the above pp-wave solution, with an NS-NS 3-form. 
On the other hand, if $d s_{6}^{2}$ is a deformed conifold, then there is a regular and supersymmetric fractional D3-brane supported by a self-dual harmonic 3-form $L_{(3)}$. This solution can be dualized to a type IIB pp-wave given by

$$
\begin{aligned}
d s_{10}^{2} & =-2 d t d x+H d x^{2}+d s_{6}^{2}+d z_{1}^{2}+d z_{2}^{2} \\
F_{(5)} & =m d x \wedge\left(d z_{1}+d z_{2}\right) \wedge L_{(3)}+\text { dual terms }
\end{aligned}
$$

\subsection{From D5-branes}

The $S^{1}$-wrapped D5-brane is given by [14

$$
\begin{aligned}
& d s_{10}^{2}=H^{-1 / 4}\left(-d t^{2}+d x_{1}^{2}+\cdots+d x_{4}^{2}+\left(d x_{5}+A_{(1)}\right)^{2}\right)+H^{3 / 4} d s_{4}^{2}, \\
& F_{(3)}^{\mathrm{RR}}=*_{4} d H-m L_{(2)} \wedge\left(d x_{5}+A_{(1)}\right), \quad \phi=-\frac{1}{2} \log H,
\end{aligned}
$$

where $d A_{(1)}=m L_{(2)} . L_{(2)}$ is a harmonic 2-form on $d s_{4}^{2}$ and $*_{4}$ is the Hodge dual with respect to $d s_{4}^{2}$. The equations of motion are satisfied, provided that

$$
\square H=-\frac{1}{4} m^{2} L_{(2)}^{2},
$$

where $\square$ is the Laplacian on $d s_{4}^{2}$. There exist regular and supersymmetric solutions for the case in which $d s_{4}^{2}$ is the metric for the Eguchi-Hanson or Taub-NUT instanton. In the case of the Eguchi-Hanson (Taub-NUT) instanton, the geometry of the D5-brane goes from a product of $E^{4,1}$ and a $U(1)$ bundle over $\mathbb{R}^{2} \times S^{2}\left(\mathbb{R}^{4}\right)$ at short distance to a domain-wall at large distance.

These solutions can be dualized to type IIB pp-waves, given by

$$
\begin{aligned}
d s_{10}^{2} & =-2 d t d x+H d x^{2}+d s_{6}^{2}+d z_{1}^{2}+d z_{2}^{2} \\
F_{(5)} & =m d x \wedge\left(d z_{1} \wedge d z_{2}+d z_{3} \wedge d z_{4}\right) \wedge L_{(2)}+\text { dual terms }
\end{aligned}
$$

We can also consider a rotating D5-brane, given by

$$
\begin{aligned}
& d s_{10}^{2}=H^{-1 / 4}\left(-\left(d t+A_{(1)}\right)^{2}+d x_{1}^{2}+\cdots+d x_{5}^{2}\right)+H^{3 / 4} d s_{4}^{2}, \\
& F_{(3)}^{\mathrm{RR}}=*_{4} d H-m L_{(2)} \wedge\left(d t+A_{(1)}\right), \quad \phi=-\frac{1}{2} \log H,
\end{aligned}
$$

where again we require the function $H$ to satisfy (5.9). This solution can be dualized to a pp-wave, given by

$$
d s_{10}^{2}=-H^{-1}\left(d t+A_{(1)}\right)^{2}+H\left(d x+\left(H^{-1}-1\right)\left(d t+A_{(1)}\right)\right)^{2}+d s_{4}^{2}+d z_{1}^{2}+\cdots+d z_{4}^{2} .
$$




\section{Conclusions}

We have constructed various supersymmetric and regular M2-brane solutions. For the transverse space, we have used eight-dimensional Ricci-flat Kähler spaces which have both non-collapsing 4-cycles and non-collapsing 2-cycles. Such spaces include a resolved cone over $Q^{1,1,1}$ and $Q^{1,1,1} / \mathbb{Z}_{2}$, as well as a product of two Eguchi-Hanson or Taub-NUT instantons. The non-collapsing 4-cycle enables the construction of a square integrable, self-dual, harmonic four-form on the transverse space, which results in a deformed M2-brane. A non-collapsing 2-cycle means that the transverse space supports a square integrable, harmonic two-form, which takes the form of a fibration in an $S^{1}$-wrapped or rotating M2-brane. Combining all of these elements into a single solution yields a deformed, $T^{2}$-wrapped, rotating M2-brane. All of the worldvolume directions, including time, are fibred over the transverse space.

Dualizing this M2-brane yields a supersymmetric and regular pp-wave in type IIB theory. Interestingly enough, in the limit in which all fluxes vanish, the geometry of the Ricci-flat pp-wave is still non-singular, due to the twisted time-like direction. Additional pp-waves of this class are obtained from wrapped and rotating D3-branes and D5-branes.

The M2-brane solutions are dual to three-dimensional $N=2$ gauge theories. The geometry of the deformed M2-brane on a resolved conifold over $Q^{1,1,1}$ smoothly goes from $E^{6,1} \times S^{2} \times S^{2}$ at short distance to $\operatorname{AdS}_{4} \times Q^{1,1,1}$ at large distance. Also, the $S^{1}$-wrapped M2-brane on a resolved conifold over $Q^{1,1,1} / \mathbb{Z}_{2}$ has a geometry that goes from a product space of $E^{1,1}$ and a $U(1)$ bundle over $\mathbb{R}^{2} \times S^{2} \times S^{2} \times S^{2}$ at short distance to $A d S_{4} \times Q^{1,1,1} / \mathbb{Z}_{2}$ at large distance. These particular solutions exhibit holographic renormalization group flows for which both conformal and Lorentz symmetries are broken in general but are restored in the UV limit. There are also cases, such as the deformed M2-brane on a resolved cone over $T^{1,1} / \mathbb{Z}_{2}$ and a deformed or wrapped M2-brane on $M_{4} \times M_{4}$, for which the geometry approaches a domain-wall at large distance and conformal symmetry is not restored in the UV limit of the dual gauge theory.

Unlike typical brane solutions which require an M-theoretic brane source term, resolved branes are complete purely within supergravity. However, not all BPS branes 
can be resolved at the level of supergravity. One benefit of $S^{1}$-wrapped $p$-branes is that they can still be non-singular even when a deformed or fractional $p$-brane on the same transverse space has a singularity. For example, a fractional D-string on a resolved cone over $Q^{1,1,1} / \mathbb{Z}_{2}$ does not have regular short-distance behavior. This is because the transverse space does not have a non-collapsing 5-cycle from which to construct a 5-form which is square integrable at short distance. It was suggested that either non-perturbative string effects were required to resolve the singularity 8 or else it is cloaked by a horizon at sufficiently high temperature 44. On the other hand, we were able to dualize our $S^{1}$-wrapped M2-brane to an $S^{1}$-wrapped D1-brane in type IIB theory, which is non-singular. This is because the transverse space does have a non-collapsing 2-cycle, which yields an appropriate fibration.

As an earlier example, we consider the resolution of the type II 5-brane. A regular heterotic or type II 5-brane wrapped around the $S^{2}$ of a resolved conifold was constructed in [47]. Alternatively, the heterotic 5-brane on an Eguchi-Hanson or Taub-NUT instanton can be resolved by making use of multiple matter Yang-Mills fields which are absent in the type II theories [11. For this reason, the analogous resolution of the type II 5-brane was unknown before the construction of the $S^{1}$-wrapped 5-brane [14, which makes use of a fibration over the transverse space.

The list of known supersymmetric and regular $S^{1}$-wrapped $p$-branes on spaces of special holonomy includes all $0 \leq p \leq 5$. However, since there are no irreducible nine and five-dimensional manifolds of special holonomy, the D0 and D4-brane must have transverse product spaces. For example, we could use $M_{n} \times S^{1}$, where $M_{n}$ is an irreducible $n$-dimensional manifold of special holonomy, and $n=8$ for the D0brane and $n=4$ for the D4-brane [11. Likewise, a regular $S^{1}$-wrapped D2-brane can be easily constructed on $M_{6} \times S^{1}$. However, a regular $S^{1}$-wrapped D2-brane on a $G_{2}$ holonomy space, if there is one with a non-vanishing 2-cycle, has yet to be constructed.

\section{ACKNOWLEDGMENT}

It is a pleasure to thank Adel Awad and Hong Lü for helpful correspondence, as well as Matthew Strassler for a useful conversation. The work of C.M.C. is supported 
in part by the National Science Council of the R.O.C. under grant number NSC922119-M-008-024. The work of J.F.V.P. is supported in part by DOE grant DE-FG0100ER45832.

\section{References}

[1] J.M. Maldacena, The large $N$ limit of superconformal field theories and supergravity, Adv. Theor. Math. Phys. 2 (1998) 231-252; Int. J. Theor. Phys. 38 (1999) 1113-1133, hep-th/9711200.

[2] S.S. Gubser, I.R. Klebanov and A.M. Polyakov, Gauge theory correlators from non-critical string theory, Phys. Lett. B 428, 105 (1998), hep-th/9802109.

[3] E. Witten, Anti-de Sitter space and holography, Adv. Theor. Math. Phys. 2, 253 (1998), hep-th/9802150.

[4] I.R. Klebanov and A.A. Tseytlin, Gravity duals of supersymmetric $S U(N) \times$ $S U(N+m)$ gauge theories, Nucl. Phys. B578 (2000) 123, hep-th/0002159.

[5] I.R. Klebanov and M.J. Strassler, Supergravity and a confining gauge theory: duality cascades and $\chi S B$-resolution of naked singularities, JHEP 0008 (2000) 052, hep-th/0007191.

[6] M. Graña and J. Polchinski, Supersymmetric three-form flux perturbations on $A d S_{5}$, Phys. Rev D63 (2001) 026001, hep-th/0009211.

[7] S. Gubser, Supersymmetry and F-theory realization of the deformed conifold with three-form flux, hep-th/0010010.

[8] M. Cvetič, G.W. Gibbons, H. Lü and C.N. Pope, Supersymmetric nonsingular fractional D2-branes and NS-NS 2-branes, Nucl. Phys. B606 (2001) 18, hep-th/0101096.

[9] P. Candelas and X.C. de la Ossa, Comments on conifolds, Nucl. Phys. B342 (1990) 246. 
[10] L.A. Pando-Zayas and A.A. Tseytlin, 3-branes on a resolved conifold, JHEP 0011 (2000) 028,hep-th/0010088.

[11] M. Cvetič, H. Lü and C.N. Pope, Brane resolution through transgression, Nucl. Phys. B600 (2001) 103, hep-th/0011023.

[12] M. Cvetič, G.W. Gibbons, H. Lü and C.N. Pope, Ricci-flat metrics, harmonic forms and brane resolutions, Commun. Math. Phys. 232 (2003) 457, hep-th/0012011.

[13] L.A. Pando-Zayas and A.A. Tseytlin, 3-branes on spaces with $R \times S^{2} \times S^{3}$ topology, Phys. Rev. D63 (2001) 086006 hep-th/0101043.

[14] H. Lü and J.F. Vázquez-Poritz, Resolution of overlapping branes, Phys. Lett. B 534155 (2002) hep-th/0202075.

[15] H. Lü and J.F. Vázquez-Poritz, $S^{1}$-wrapped D3-branes on conifolds, Nucl. Phys. B 633114 (2002), hep-th/0202175.

[16] M. Cvetič, G.W. Gibbons, H. Lü and C.N. Pope, M-theory conifolds, Phys. Rev. Lett. 88 (2002) 121602, hep-th/0112098.

[17] A. Brandhuber, $G_{2}$ holonomy spaces from invariant three-forms, Nucl. Phys. B629 (2002) 393, hep-th/0112113.

[18] M. Cvetič, G.W. Gibbons, H. Lü and C.N. Pope, A $G_{2}$ unification of the deformed and resolved conifolds, Phys. Lett. B534 (2002) 172, hep-th/0112138.

[19] M.J. Duff, H. Lü, C.N. Pope and E. Sezgin, Supermembranes with fewer supersymmetries, Phys. Lett. B371 (1996) 206, hep-th/9511162.

[20] K. Becker and M. Becker, M-theory on eight-manifolds, Nucl. Phys. B477 (1996) 155, hep-th/9605053.

[21] M.J. Duff, J.M. Evans, R.R. Khuri, J.X. Lu and R. Minasian, The octonionic membrane, Phys. Lett. B412 (1997) 281, hep-th/9706124.

[22] S.W. Hawking and M.M. Taylor-Robinson, Bulk charges in eleven dimensions, Phys. Rev. D58 (1998) 025006, hep-th/9711042. 
[23] K. Becker, A note on compactifications on spin(7) manifolds, JHEP 0105 (2001) 003, hep-th/0011114.

[24] C.P. Herzog and I.R. Klebanov, Gravity duals of fractional branes in various dimensions, Phys. Rev. D63 (2001) 126005, hep-th/0101020

[25] M. Cvetič, G.W. Gibbons, H. Lü and C.N. Pope, Hyper-Kähler Calabi metrics, $L^{2}$ harmonic forms, resolved M2-branes, and $A d S_{4} / C F T_{3}$ correspondence, Nucl. Phys. B617 (2001) 151, hep-th/0102185.

[26] M. Cvetič, G.W. Gibbons, H. Lü and C.N. Pope, New complete non-compact Spin(7) manifolds, Nucl. Phys.B620 (2002) 29, hep-th/0103155.

[27] M. Cvetič, G.W. Gibbons, J.T. Liu, H. Lü and C.N. Pope, A new fractional D2-brane, $G_{2}$ holonomy and T-duality, Class. Quant. Grav. 19 (2002) 5163, hep-th/0106162.

[28] S. Gukov and J. Sparks, M-theory on Spin(7) manifolds, Nucl. Phys. B625 (2002) 3, hep-th/0109025.

[29] F.A. Brito, M. Cvetič and A. Naqvi, Brane resolution and gravitational ChernSimons terms, Class. Quant. Grav. 20 (2003) 285, hep-th/0206180.

[30] J.P. Gauntlett and S. Pakis, The geometry of D $=11$ killing spinors, JHEP 0304 (2003) 039, hep-th/0212008

[31] N. Khviengia, Z. Khviengia, H. Lü and C.N. Pope, Towards a field theory of F-theory, Class. Quant. Grav. 15 (1998) 759, hep-th/9703012.

[32] L. Castellani, L.J. Romans and N.P. Warner, A classification of compactifying solutions for $d=11$ supergravity, Nucl. Phys. B241 (1984) 429.

[33] T. Eguchi and A.J. Hanson, Asymptotically flat self-dual solutions to Euclidean gravity, Phys. Lett. B74 (1978) 249.

[34] S.W. Hawking, Gravitational instantons, Phys. Lett. A60 (1977) 81. 
[35] B.S. Acharya, J.M. Figueroa-O'Farrill, C.M. Hull and B. Spence, Branes at conical singularities and holography, Adv. Theor. Math. Phys. 2 (1999) 1249, hep-th/9808014.

[36] D.R. Morrison and M.R. Plesser, Non-spherical horizons. I, Adv. Theor. Math. Phys. 3 (1999) 1, hep-th/9810201.

[37] K. Oh and R. Tatar, Three dimensional SCFT from M2 branes at conifold singularities, JHEP 9902 (1999) 025, hep-th/9810244.

[38] G. Dall'Agata, $N=2$ conformal field theories from M2-branes at conifold singularities, Phys. Lett. B460 (1999) 79, hep-th/9904198.

[39] D. Fabbri, P. Fre, L. Gualtieri and P. Termonia, Osp $(\mathrm{N}-4)$ supermultiplets as conformal superfields on $d(A d S(4))$ and the generic form of $N=2, D=3$ gauge theories, Class. Quant. Grav. 17 (2000) 55, hep-th/9905134.

[40] C.h. Ahn, $N=2$ SCFT and M-theory on $\operatorname{AdS}(4) \times Q(1,1,1)$, Phys. Lett. B466 (1999) 171, hep-th/9908162

[41] D. Fabbri, Three dimensional conformal field theories from Sasakian sevenmanifolds, hep-th/0002255.

[42] U. Gursoy, C. Nunez and M. Schvellinger, RG flows from Spin(7), CY 4-fold and HK manifolds to AdS, Penrose limits and pp waves, JHEP 0206 (2002) 015, hep-th/0203124.

[43] R. Hernandez and K. Sfetsos, Branes with fluxes wrapped on spheres, JHEP 0207 (2002) 045, hep-th/0205099

[44] C.P. Herzog and P. Ouyang, Fractional D1-branes at finite temperature, Nucl. Phys. B610 (2001) 97, hep-th/0104069.

[45] M.J. Duff, H. Lü and C.N. Pope, $A d S_{5} \times S^{5}$ untwisted, Nucl. Phys. B532 (1998) 181, hep-th/9803061

[46] M. Cvetič, H. Lü and C.N. Pope, Penrose limits, pp-waves and deformed M2branes, hep-th/0203082. 
[47] J.M. Maldacena and C. Núnez, Towards the large $N$ limit of pure $\mathcal{N}=1$ super Yang Mills, Phys. Rev. Lett. 86 (2001) 588, hep-th/0008001. 